\title{
Editorial
}

\section{Acknowledgement to Reviewers of Axioms in 2014}

Axioms Editorial Office, MDPI AG, Klybeckstrasse 64, CH-4057 Basel, Switzerland

Published: 8 January 2015

The editors of Axioms would like to express their sincere gratitude to the following reviewers for assessing manuscripts in 2014:

Alagic, Gorjan

Alecsandri, Vasile

Ali, S. Twareque

Andeichicova, Olga

Baseilhac, Pascal

Begum, Shahina

Bernardino, Alexandre

Bouret, Yann

Bronstein, Alex

Brun, Luc

Brzezinski, Tomasz

Calin, Ovidiu

Chan, Hei-Chi

Chipchakov, Ivan

Cobre, Juliana

Courty, Nicolas

de Gosson, Maurice

Deriche, Rachid

Donoho, David

Dürr, Detlef

Ernst, Thomas

Falush, Daniel

Feret, Jérôme

Fine, Arthur
Freyd, Peter

Gaüzère, Benoit

Gudder, Stanley

Guirao, Juan L. G.

Gur, Yaniv

Hall, Michael

He, Ying

Ishizaka, Alessio

Jin, Miao

Johnson, Niles

Jorgensen, Palle

Jost, Jürgen

Kastner, Ruth

Kim, Song-Kyoo

Korepin, Vladimir E.

Lahti, Pekka

Liu, Shiping

López, Luis Martínez

Luo, Jun

Mainardi, Francesco

Malley, James

Marcus, Solomon

Martin, Clare

Mérigot, Quentin
Metzler, Ralf

Mori, Ryuhei

Morigi, Serena

Morita, Takeshi

Nieto, Juan J.

Nocera, Luigi

Pagnini, Gianni

Pereira, Ricardo Alberto

Marques

Pinyol, Isaac

Postolica, Vasile

Povstenko, Yuriy

Quarati, P.

Rathjen, Michael

Santos, Emilio

Saucan, Emil

Sbitnev, Valery

Sivasundaram, Seenith

Solovjovs, Sergejs

Srivastava, Hari M.

Stern, Julio Michael

Street, Ross

Sudarsky, Daniel

Sur, Frédéric 
Velickovic, Boban

Viana, M

Vicente, Renato

Vourdas, Apostolos

Wan, Shenghua
Wetterich, Christof

Whitaker, Rozann

Whitfield, James Daniel

Winter, Michael

Wirth, Benedikt
Wüthrich, Christian

Zayed, Ahmed

Zeng, Wei

Zhang, Junping

(C) 2015 by the authors; licensee MDPI, Basel, Switzerland. This article is an open access article distributed under the terms and conditions of the Creative Commons Attribution license (http://creativecommons.org/licenses/by/4.0/). 